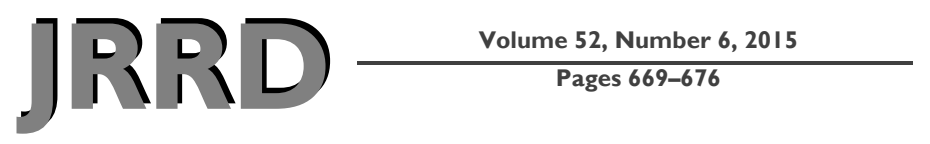

\title{
Traumatic brain injury in U.S. Veterans with traumatic spinal cord injury
}

\author{
Graham H. Creasey, MD; ${ }^{1-2^{*}}$ Zoia C. Lateva, PhD; ${ }^{1}$ Sophia Miryam Schüssler-Fiorenza Rose, MD, PhD; ${ }^{1-2}$ \\ Jon Rose, PhD ${ }^{1}$ \\ ${ }^{1}$ Spinal Cord Injury and Disorders Center, Department of Veterans Affairs Palo Alto Health Care System, Palo Alto, \\ CA; ${ }^{2}$ Department of Neurosurgery, Stanford University School of Medicine, Stanford, CA
}

\begin{abstract}
Patients with both a spinal cord injury (SCI) and traumatic brain injury (TBI) are often very difficult to manage and can strain the resources of clinical units specialized in treating either diagnosis. However, a wide range of estimates exists on the extent of this problem. The aim of this study was to describe the scope of the problem in a well-defined population attending a comprehensive SCI unit. Electronic medical records of all patients with SCI being followed by the SCI unit in a U.S. Veterans' hospital were searched to identify those with concurrent TBI. The data were analyzed for age, sex, cause of injury, level and completeness of SCI, cognitive impairment, relationship with Active Duty military, and date of injury. Of 409 Veterans with a traumatic SCI, 99 (24.2\%) were identified as having had a concurrent TBI. The occurrence did not appear to be closely related to military conflict. Reports of TBI were much more common in the last 20 yr than in previous decades. Documentation of TBI in patients with SCI was inconsistent. Improved screening and documentation could identify all patients with this dual diagnosis and facilitate appropriate management.
\end{abstract}

Key words: Active Duty military, cognitive impairment, electronic medical records, military trauma, polytrauma, retrospective study, spinal cord injury, traumatic brain injury, Veterans, Veterans Health Administration.

\section{INTRODUCTION}

Since World War II, when patients with spinal cord injury (SCI) began to survive in significant numbers, it has been recognized that SCI is often associated with other injuries. Recent military conflicts have drawn renewed attention to traumatic brain injury (TBI) and SCI [1-2]. Both can be catastrophic, and their management is particularly difficult when they occur in the same individual. Patients with paraplegia and co-occurring severe TBI have been shown to have worse motor outcomes and longer acute rehabilitation lengths of stay than those with paraplegia and no TBI [3]. There is, however, a wide range of estimates of the prevalence of such a dual diagnosis [4].

The prevalence of SCI among patients with TBI is relatively low. Two decades ago, acute spinal cord trauma was estimated to occur in between 5 and 15 percent of patients with severe head injury [5-6]. A recent article on closed or penetrating head injury sustained in military personnel in Iraq reported a 9.8 percent incidence of SCI or spinal column injury [7]. A retrospective study of 447 patients with moderate or severe head injury evaluated at two civilian level 1 trauma centers showed that 3.1 percent sustained SCI [8]. A prospective study of 180 patients with moderate or severe TBI admitted to a neurotrauma intensive care unit found that 7.8 percent had SCI [9].

Abbreviations: CPRS = Computerized Patient Record System, ICD-9-CM = International Classification of Diseases-9th Revision-Clinical Modification, $\mathrm{SCI}=$ spinal cord injury, TBI = traumatic brain injury, VA = Department of Veterans Affairs.

*Address all correspondence to Graham $\mathrm{H}$. Creasey, MD; VA Palo Alto Health Care System, 3801 Miranda Ave, Room C115A, Building 7, Palo Alto, CA 94304; 650-7042394; fax: 650-849-1238. Email: graham.creasey@va.gov http://dx.doi.org/10.1682/JRRD.2014.11.0291 
The prevalence of TBI among patients with SCI is not clear and has a wide range of estimates. The Spinal Cord Injury Model Systems reported retrospectively in 1995 that 28.2 percent of patients with SCI had at least a mild TBI with loss of consciousness and 11.5 percent had a TBI severe enough to demonstrate cognitive or behavioral changes [10]. A more recent prospective study of a sample of 198 patients with SCI in a single large SCI model system estimated that 60 percent had TBI [4]. There may be several reasons for uncertainty about the prevalence of TBI in patients with SCI. The location of the study (e.g., trauma service, intensive care unit, SCI unit) and whether the injuries occurred in a military or civilian context will determine the population studied. Much depends on the design of the study and definitions used for TBI, particularly mild TBI whose definition has not always been clear and which can be confused with posttraumatic stress disorder and other conditions. Patients themselves may underreport TBI through lack of insight or a desire to return to Active Duty or overreport TBI in the context of compensation or to avoid the stigma of mental illness [11-13]. TBI may be particularly underdiagnosed in retrospective studies [14], where it is highly dependent on documentation.

In view of the wide range of published estimates and the medical, social, and financial significance of these injuries, we investigated documentation of TBI in a welldefined population with known SCI. The U.S. Department of Veterans Affairs (VA) has a national System of Care for Spinal Cord Injuries and Disorders, with 24 SCI centers and 127 SCI clinics arranged in a hub and spoke system. Veterans of the U.S. military with spinal cord injuries or disorders are accepted into this system for rehabilitation and lifetime follow-up, and their medical records are maintained in an electronic medical record known as the Computerized Patient Record System (CPRS). Most of the contents of this system are textbased, but they can be searched electronically, allowing for the examination of thousands of notes extending over many years.

\section{METHODS}

The SCI service of the VA Palo Alto Health Care System maintains a registry of patients with spinal cord injuries or disorders that undergo initial inpatient rehabilitation or follow-up care. Some of these patients sus- tained their SCI many years ago and continue to receive active follow-up at the SCI service as is recommended by the VA. The current study included patients who were admitted initially or for follow-up care during a 2 yr period (October 2010-October 2012). Patients who died during this period were not excluded. Since our interest was in co-occurrence of SCI and TBI, the first step was to identify the subset of patients with traumatic SCI. The etiology of traumatic SCI was determined using the Common Data Elements classification of (1) sports and leisure, (2) assault, (3) transport, (4) fall, and (5) other traumatic causes [15]. The Common Data Elements classification is based on the World Health Organization International Classification of External Causes of Injury and recommends that when the etiology is classifiable into more than one of these five categories, the category with the highest priority (the lowest number) should be assigned.

The personal identifiers of these patients were then used to obtain their electronic medical records in the VA CPRS and search for any reference to TBI or head injury. The electronic medical record of each patient includes a list of Active Problems, which uses codes of the International Classification of Diseases-9th Revision-Clinical Modification (ICD-9-CM). The Active Problems list was examined and if a reference to TBI or head injury was found, the ICD-9-CM code was noted as well as the date of entry, which was used to determine whether the injury happened at the same time as the SCI. The electronic medical record may contain hundreds or even thousands of notes for each patient. The text of all notes was searched electronically for any of the following text phrases: "TBI," "brain injury," "brain trauma," "head injury," "head trauma," "loss of consciousness," or "LOC." When any of these words or phrases was found in the notes, their context was examined to determine whether the patient did indeed have a history of any TBI (mild, moderate, severe, or self-reported) and its relationship in time to the SCI. For patients identified as having TBI that occurred at the same time as SCI, an additional search for cognitive impairment was conducted. SCI service psychologists perform an annual evaluation of all patients. Every note from the SCI service psychologists includes a section on cognitive functioning that was identified by this search; each of these notes was then read to determine whether there was cognitive impairment and whether it was attributable to the TBI or to other known conditions. Other information was also extracted from 
the electronic medical record, such as sex, current age, age at the time of the SCI, date of SCI, and level and completeness of the spinal cord lesion. The military status (Active Duty or retired) of the Veteran at the time of the injury was also determined.

\section{RESULTS}

A total of 701 patients were included in this study, of whom 675 (96.3\%) were male and 26 (3.7\%) were female, which is typical in the Veteran population with SCI. Screening by cause of spinal cord or column damage revealed that 292 patients had nontraumatic damage and were excluded from further analysis. The remaining 409 (58\%) patients had traumatic SCI, of whom only 9 were female. The mean \pm standard deviation current age of patients with traumatic SCI was $60 \pm 13$ yr (range: 23$98 \mathrm{yr}$ ) and the mean time since injury was $24 \pm 16 \mathrm{yr}$ (range: 1-69 yr).
Of the 409 patients with traumatic SCI, only 18 had any reference to TBI noted in their Active Problem list; in all these cases, the TBI had occurred at the same time as the SCI. However, electronic searching of the text of notes using the criteria described in the "Methods" section identified an additional 81 patients with traumatic SCI as having had a TBI at the same time as the SCI. Thus, a total of 99 out of 409 patients with traumatic SCI (24.2\%) had experienced a concurrent TBI.

The Active Problems list in the CPRS thus did not record the existence of the TBI in over 80 percent of these cases. In many of these cases, the head and/or brain injury or trauma was also not mentioned in the history recorded on admission but only in the text of notes by psychologists working in the SCI service, sometimes when reviewing a patient years after the injury.

The available information was not sufficient to determine the severity of the TBI in each case. Table 1 shows the distribution by sex and age group. Table 1 also shows the distribution and frequencies by etiology of the traumatic SCI. Transport (category 3) was the most common

Table 1.

Distribution and frequencies (\%) of key patient characteristics by group: concurrent traumatic brain injury (TBI) and spinal cord injury (SCI), SCI only, and SCI total.

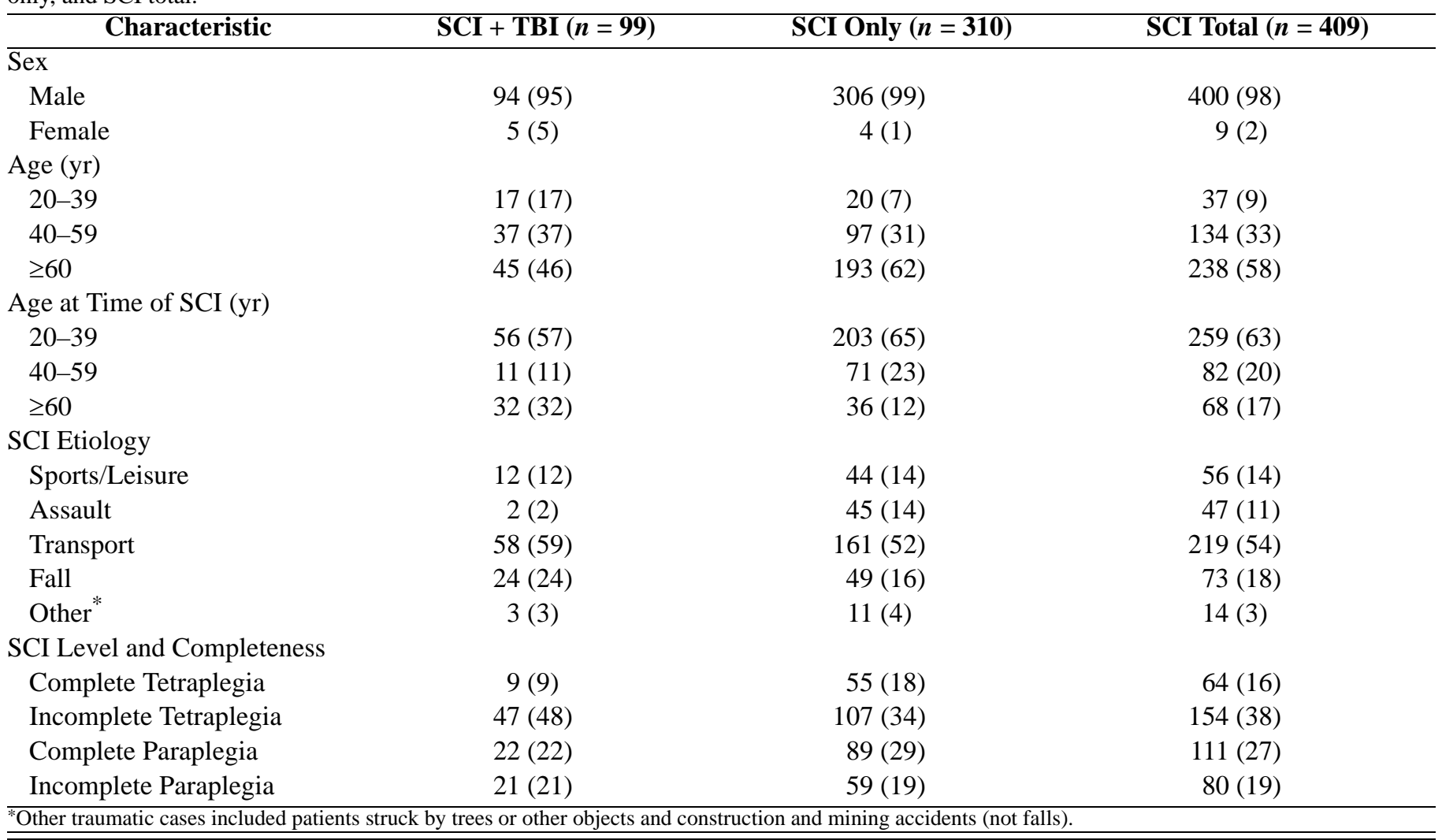


cause both in patients with SCI only (52\%) and those with concurrent SCI and TBI (59\%). Fall (category 4) was the second most common cause. Notably, when the SCI was caused by assault (category 2), concurrent TBI was rare. Of 47 cases with SCI due to assault, 39 were due to gunshot wounds and none of these 39 had concurrent TBI. The two cases with concurrent SCI and TBI due to assault were caused by shrapnel and rocket-propelled grenade, respectively. Thus, only 4 percent of all assault cases led to concurrent SCI and TBI compared with 26.8 percent of nonassault SCI etiologies.

The number of patients with cervical SCI was only slightly higher than the number with thoracic and lumbar SCI: 218 (53.3\%) tetraplegia versus 191 (46.7\%) paraplegia (Table 1). The frequencies of concurrent SCI and TBI among tetraplegia patients (25.7\%) and among paraplegia patients (22.5\%) were also similar.

About half of the patients with concurrent traumatic SCI and TBI were also found to have cognitive impairment: 54 out of 99 (55\%). Further comments on this finding are given in the "Discussion" section.

Investigation of when the concurrent SCI and TBI occurred in relation to military service (Active Duty or after military discharge) revealed that most of the patients (69 [70\%]) had been injured after leaving military duty. While 30 of the injuries occurred during Active Duty, the majority of cases (20) were caused by accidents during transport by road or air, 8 were caused by falls and sport or leisure activities, and only 2 were caused by assault (shrapnel or rocket-propelled grenade).

When patients were stratified according to the date of their traumatic SCI, it became notable that records of concurrent TBI had increased greatly in recent years (Table 2). Half of all patients for whom we found electronic medical records of concurrent SCI and TBI were injured in the last decade (2003 to 2012). For comparison, the cases with SCI were more evenly distributed
(15\% to $24 \%$ ) between different decades. Possible reasons for this are discussed later.

\section{DISCUSSION}

In this single-center retrospective study, the overall percentage of patients with traumatic SCI who were recorded as having had a concurrent TBI was 24.2 percent, and for nonassault etiologies the frequency was slightly higher (26.8\%). This frequency is similar to multicenter retrospective figures from the SCI model systems reported in 1995 (28.2\%) [10] but lower than figures from a prospective study in a single SCI model system reported in 2008 (60\%) [4]. We believe that the higher frequency found in the prospective research study is attributable to more accurate screening and documentation of associated injuries at the time of the SCI and that more consistent screening and documentation of associated injuries, particularly TBI, is required in clinical practice, as discussed later.

Some studies have found cervical SCI to be associated with greater rates of concurrent TBI $[4,9]$. Our results did not show significant differences in the frequency of TBI between the groups of patients with tetraplegia or paraplegia (Table 1).

In the current study, the number of Veterans with traumatic SCI in whom a concurrent TBI was recorded has increased substantially over recent decades, from $<9$ percent before 1983 to 50 percent since 2003, while the number of patients with SCI only stayed more or less similar over time (Table 2). Three possible reasons are discussed next.

\section{Military Activity}

In recent years, TBI has been described as the signature injury of military action in the Middle East, and it might be hypothesized that this has increased the number

Table 2.

Distribution and frequencies (\%) of concurrent traumatic brain injury (TBI) and spinal cord injury (SCI), SCI only, and SCI total by date of injury.

\begin{tabular}{lccr}
\hline Date of Injury & SCI + TBI $(\boldsymbol{n}=\mathbf{9 9})$ & SCI Only $(\boldsymbol{n}=\mathbf{3 1 0})$ & SCI Total $(\boldsymbol{n}=\mathbf{4 0 9})$ \\
\hline $2003-2012$ & $49(50)$ & $71(23)$ & $120(29)$ \\
$1993-2002$ & $23(23)$ & $47(15)$ & $70(17)$ \\
$1983-1992$ & $11(11)$ & $50(16)$ & $61(15)$ \\
$1973-1982$ & $6(6)$ & $74(24)$ & $80(20)$ \\
$1963-1972$ & $9(9)$ & $56(18)$ & $65(16)$ \\
$<1963$ & $1(1)$ & $12(4)$ & $13(3)$ \\
\hline \hline
\end{tabular}


of patients with SCI and TBI. However, patients whose SCI was caused by gunshot wound or shrapnel were rarely recorded in this study as having a TBI, presumably because missiles strike either the spine or head but rarely both, and in recent conflicts the use of body armor appears to have greatly reduced the incidence of SCI. It is well known that during Active Duty, many injuries are caused not in combat but by other forms of trauma such as motor vehicle accidents. In this study, while 30 percent of concurrent injuries occurred during Active Duty, the majority of these occurred during transport by road or air. Only 7 of 99 concurrent injuries occurred during combat, and none were caused by a gunshot; one was caused by shrapnel, one by rocket-propelled grenade, two by motor vehicle accidents, and three by flying accidents. It seems likely, therefore, that the contribution of military combat to increasing records of concurrent SCI and TBI is small.

\section{Improved Documentation}

The CPRS was introduced by the VA in 1997. Patients injured before this time have their current medical records entered into this system, but the notes about their medical history before this time are often dependent on patients' memory, which can be impaired if they had a head injury or because of their current age. The CPRS provides improved ability to retrieve information entered about injuries since 1997. However, proper documentation also depends on whether patients are asked about the possibility of past head injury even if the injury resolved, which depends on awareness by clinical staff of the possibility of head injury.

\section{Improved Awareness}

Awareness of head injury in military personnel has increased during the last two decades, and this has led to increased screening in the Department of Defense and VA. Psychologists working in SCI units are usually aware of this, but other staff, including medical residents in training who may do much of the documentation, may be less aware of the possibility of head injury and less skilled in diagnosing it. The results of screening in the Department of Defense are not necessarily made available to the VA when a patient is transferred. We believe that more consistent documentation and communication of this information should become standard practice in the Department of Defense and VA to reduce the chance of missing a diagnosis of TBI.
Traumatic SCI usually has obvious symptoms and signs and is therefore relatively rarely missed, and major TBI is rarely missed as well. When both are present, management is usually assigned to either an SCI or TBI unit, depending on the relative severity of the two injuries. Ideally, the staff of such units would collaborate in the management of such patients. However, SCI and TBI units may not be located in the same institution, and even when they are, they often have different cultures and collaboration may be limited. In practice, each unit will concentrate on the injury it knows best, and the other injury may not receive state-of-the-art attention.

Less severe TBI can be missed, particularly in patients with multiple and life-threatening injuries who may be in shock, undergoing emergency surgery, sedated, or on a ventilator. When they are stabilized, their management will depend somewhat on the service to which they are transferred and on its awareness of the possibility of concurrent injuries.

Identification of TBI in electronic medical records of patients with SCI in this study was inconsistent. It might be thought that this was because the head injury was mild in this series of patients, but 55 percent of the Veterans with concurrent TBI and SCI were identified as having cognitive impairment. This is similar to the percentage found in patients with SCI treated in the SCI model systems of care. While cognitive impairment can be due to causes other than TBI in these patients, it remains important to identify whether they have had a TBI. During annual evaluations of Veterans with SCI, our VA SCI service psychologists evaluate attention, problem solving, processing speed, and memory by interview, and if cognitive impairment is suspected they supplement the interview with neuropsychological tests selected on the basis of the impairment suspected. The most frequent terms used in the electronic medical record for cognitive impairment were slowed processing speed and "shortterm memory loss," although it is now recognized that it would be beneficial to use clearer terms to describe attention and memory in the medical record. In the past, the term short-term memory loss used in the medical record has often been nonspecific and may have been used to refer to anything from working memory of a few seconds to recall of the events from a few weeks before.

In the case of patients with mild TBI, it might have been argued in the past that they did not suffer greatly from delayed or absent documentation of the injury, but there is now increased interest in the unknown long-term 
effects of mild and repeated TBI on conditions such as Parkinson disease and dementia. The fact that the VA healthcare system follows patients with SCI for life offers an opportunity to study the relationship between these conditions.

The use of an electronic medical record in the VA has had many advantages, but it may be necessary to structure the collection and recording of some information in a more consistent way that could be implemented in a national system of care. Consistent screening of patients with SCI for TBI during their initial rehabilitation should be done to avoid missing the diagnosis of TBI. If TBI resolves, there is no way to identify it subsequently other than history from the patient, collaterals, and prior medical reports. Screening will need to be done after patients are stabilized on medications for pain and spasticity since these medications are known to affect cognitive functioning until patients accommodate to them. Patients will also need to be clear of delirium from surgical anesthesia, urinary tract infections, and other SCI complications. In many cases, it will be difficult to distinguish TBI from depression, posttraumatic stress disorder, and/or anxiety, so diagnosis will be delayed until psychiatric symptoms are adequately treated. These are some of many reasons for providing adequate time for rehabilitation rather than discharging patients as soon as they can survive outside of a hospital. Fortunately, adequate admission time is standard practice in the VA. It is possible that our finding that 50 percent of Veterans who experienced traumatic SCI in the last $10 \mathrm{yr}$ are currently being recorded as having a concurrent TBI is still an underestimate, so there may still be a significant number of Veterans in whom TBI has not been diagnosed as suggested by the recent study in civilians [4]. It is critically important to avoid missing the diagnosis of TBI in Veterans (and others) if appropriate rehabilitation and follow-up care is to be provided.

The causes of SCI in Veterans with concurrent TBI, such as motor vehicle accidents, falls, and sporting accidents, resemble the causes seen in the civilian population, so some of the conclusions of this study may be applied to civilians. It would be of value to improve documentation of TBI in the civilian population with SCI, particularly as electronic medical records are being adopted. This could best be done within the SCI model systems, even though only a minority of U.S. civilians with SCI receives their care in these systems and they may have a higher proportion of patients with severe SCI than in the population treated outside the SCI model systems. Such documentation would allow for the collection of data in civilians comparable to that in Veterans and provide guidelines for future management of long-term consequences of these injuries, especially in young people.

\section{CONCLUSIONS}

1. Documentation of TBI in this population of Veterans with traumatic SCI was inconsistent; in patients with both SCI and TBI, the TBI identified by searching the notes in the electronic medical record was only recorded in the Active Problems list 18 percent of the time and was often absent from admission histories and discharge summaries.

2. Recorded incidents of TBI in Veterans with traumatic SCI in this study have increased from $<9$ percent before 1983 to 50 percent since 2003. This may reflect improved documentation and increased awareness, but there may be more cases that are still not being identified. Extrapolation of these figures nationally suggests that there may be a substantial number of Veterans whose TBI has not been documented.

3. Improved screening and documentation would help to identify all Veterans with both SCI and TBI and allow appropriate management and long-term follow-up.

4. Based on these findings, we propose the following recommendations:

a. Screening by a psychologist of all Veterans newly enrolled in the VA System of Care for Spinal Cord Injuries and Disorders to assess for potential TBI, because all VA SCI centers are required to have a psychologist in their team.

b. Structuring documentation of TBI in the CPRS at least in the Active or Inactive Problems lists and perhaps in other locations and templates.

c. Making available the baseline automated neuropsychological assessment data now collected on all U.S. military personnel to VA clinicians in the VA CPRS and using these data to assist in screening all patients with SCI for TBI when they are enrolled in the VA System of Care for Spinal Cord Injuries and Disorders.

d. Training of medical residents, fellows, and other physicians who work with SCI to screen for TBI.

e. Screening for TBI in non-Veteran populations with traumatic SCI, with documentation led by the SCI 
model systems and their database in the National Spinal Cord Injuries Statistical Center.

\section{ACKNOWLEDGMENTS}

\author{
Author Contributions: \\ Study concept and design: G. H. Creasey. \\ Acquisition of data: Z. C. Lateva. \\ Analysis and interpretation of data: G. H. Creasey, Z. C. Lateva, \\ J. Rose, S. M. Schüssler-Fiorenza Rose. \\ Drafting of manuscript: G. H. Creasey, Z. C. Lateva. \\ Critical revision of manuscript for important intellectual content: \\ J. Rose, S. M. Schüssler-Fiorenza Rose. \\ Obtained funding: G. H. Creasey. \\ Administrative, technical, or material support: G. H. Creasey.
}

Financial Disclosures: The authors have declared that no competing interests exist.

Funding/Support: This material was based on work supported by a Translational Research Partnership Award (no. W81XWH-10-10911) from the U.S. Department of Defense Spinal Cord Injury Research Program (grant SC090241). Dr. Schüssler-Fiorenza Rose was supported by the VA Advanced Fellowship Program in Spinal Cord Injury Medicine.

Additional Contributions: The authors would like to acknowledge Michael Beattie, $\mathrm{PhD}$, and Jacqueline Bresnahan, $\mathrm{PhD}$, of the Brain and Spinal Injury Center at the University of California San Francisco who initiated the Translational Research Partnership Award funded by the Department of Defense. We wish to thank all the members of the Translational Research Partnership for their productive discussions and collaboration, in particular Stephen McKenna, MD, of the Rehabilitation Trauma Center and the Spinal Cord Injury Unit at the Santa Clara Valley Medical Center in San Jose, California.

Institutional Review: The study was approved by the institutional review board of Stanford University on behalf of the VA Palo Alto Health Care System.

\section{REFERENCES}

1. Galarneau MR, Woodruff SI, Dye JL, Mohrle CR, Wade AL. Traumatic brain injury during Operation Iraqi Freedom: Findings from the United States Navy-Marine Corps Combat Trauma Registry. J Neurosurg. 2008;108(5):950-57. [PMID:18447712] http://dx.doi.org/10.3171/JNS/2008/108/5/0950

2. Schoenfeld AJ, Sielski B, Rivera KP, Bader JO, Harris MB. Epidemiology of cervical spine fractures in the US military. Spine J. 2012;12(9):777-83. [PMID:21393068] http://dx.doi.org/10.1016/j.spinee.2011.01.029

3. Macciocchi S, Seel RT, Warshowsky A, Thompson N, Barlow K. Co-occurring traumatic brain injury and acute spinal cord injury rehabilitation outcomes. Arch Phys Med
Rehabil. 2012;93(10):1788-94. [PMID:22480549]

http://dx.doi.org/10.1016/j.apmr.2012.01.022

4. Macciocchi S, Seel RT, Thompson N, Byams R, Bowman B. Spinal cord injury and co-occurring traumatic brain injury: Assessment and incidence. Arch Phys Med Rehabil. 2008;89(7):1350-57. [PMID:18586138] http://dx.doi.org/10.1016/j.apmr.2007.11.055

5. Hills MW, Deane SA. Head injury and facial injury: Is there an increased risk of cervical spine injury? J Trauma. 1993;34(4):549-53, discussion 553-54. [PMID:8487340] http://dx.doi.org/10.1097/00005373-199304000-00011

6. Michael DB, Guyot DR, Darmody WR. Coincidence of head and cervical spine injury. J Neurotrauma. 1989;6(3): 177-89. [PMID:2810382] http://dx.doi.org/10.1089/neu.1989.6.177

7. Bell RS, Vo AH, Neal CJ, Tigno J, Roberts R, Mossop C, Dunne JR, Armonda RA. Military traumatic brain and spinal column injury: A 5-year study of the impact blast and other military grade weaponry on the central nervous system. J Trauma. 2009;66(4 Suppl):S104-11.

[PMID:19359953]

http://dx.doi.org/10.1097/TA.0b013e31819d88c8

8. Holly LT, Kelly DF, Counelis GJ, Blinman T, McArthur DL, Cryer HG. Cervical spine trauma associated with moderate and severe head injury: Incidence, risk factors, and injury characteristics. J Neurosurg. 2002;96(3 Suppl):285-91.

[PMID:11990836]

9. Paiva WS, Oliveira AM, Andrade AF, Amorim RL, Lourenço LJ, Teixeira MJ. Spinal cord injury and its association with blunt head trauma. Int J Gen Med. 2011;4:613-15.

[PMID:21941446]

http://dx.doi.org/10.2147/IJGM.S15811

10. Go BK, De Vivo MJ, Richards DS. The epidemiology of spinal cord injury. In: Stover SL, DeLisa JA, Whiteneck GG, editors. Spinal cord injury: Clinical outcomes from the model systems. Gaithersburg (MD): Aspen; 1995. p. 21-51.

11. Tolonen A, Turkka J, Salonen O, Ahoniemi E, Alaranta H. Traumatic brain injury is under-diagnosed in patients with spinal cord injury. J Rehabil Med. 2007;39(8):622-26.

[PMID:17896053]

http://dx.doi.org/10.2340/16501977-0101

12. Prigatano GP. Behavioral limitations TBI patients tend to underestimate: A replication and extension to patients with lateralized cerebral dysfunction. Clin Neuropsychol. 1996; 10(2):191-201. http://dx.doi.org/10.1080/13854049608406680

13. Drake AI, Meyer KS, Cessante LM, Cheung CR, Cullen MA, McDonald EC, Holland MC. Routine TBI screening following combat deployments. NeuroRehabilitation. 2010; 26(3):183-89. [PMID:20448308] http://dx.doi.org/10.3233/NRE-2010-0554 
14. Snell DL, Siegert RJ, Hay-Smith EJ, Surgenor LJ. Associations between illness perceptions, coping styles and outcome after mild traumatic brain injury: Preliminary results from a cohort study. Brain Inj. 2011;25(11):1126-38. [PMID:21870903] http://dx.doi.org/10.3109/02699052.2011.607786

15. Biering-Sørensen F, Charlifue S, Devivo MJ, Grinnon ST, Kleitman N, Lu Y, Odenkirchen J. Using the spinal cord injury common data elements. Top Spinal Cord Inj Rehabil. 2012;18(1):23-27. [PMID:22408366]

http://dx.doi.org/10.1310/sci1801-23

Submitted for publication November 20, 2014. Accepted in revised form May 5, 2015.

This article and any supplementary material should be cited as follows:
Creasey GH, Lateva ZC, Schüssler-Fiorenza Rose SM, Rose J. Traumatic brain injury in U.S. Veterans with traumatic spinal cord injury. J Rehabil Res Dev. 2015;52(6): 669-760.

http://dx.doi.org/10.1682/JRRD.2014.11.0291

ResearcherID/ORCID: Graham H. Creasey, MD: J-61362015/0000-003-1720-4491; Zoia C. Lateva, PhD: J6142-2015; Sophia Miryam Schüssler-Fiorenza Rose, MD, PhD: 0000-0002-6311-6671; Jon Rose, PhD: J6141-2015/0000-0003-3350-5526

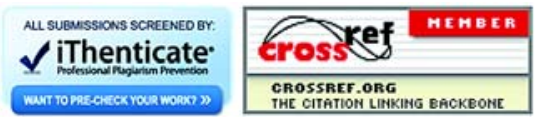

